\title{
ADAPTING PETRI NETS TO DES: STOCHASTIC MODELLING OF MANUFACTURING SYSTEMS
}

\author{
Simon, E. ; Oyekan, J. ${ }^{* *}$; Hutabarat, W. ${ }^{* * *}$; Tiwari, A. ${ }^{* * *} \&$ Turner, C. J.,\# \\ *Manufacturing Department, Cranfield University, Bedfordshire, MK43 0AL, UK \\ ** Intelligent Automation and Informatics Theme, The Manufacturing Technology Centre, Ansty Park, \\ Coventry, CV7 9JU, UK \\ ${ }^{* * *}$ The University of Sheffield, Department of Automatic Control and Systems Engineering (ACSE), \\ Sheffield, S1 3JD, UK \\ E-Mail: e.simon@cranfield.ac.uk,john.oyekan@the-mtc.org,w.hutabarat@sheffield.ac.uk, \\ a.tiwari@ sheffield.ac.uk, c.j.turner@cranfield.ac.uk ( ${ }^{\#}$ Corresponding author)
}

\begin{abstract}
Discrete-Event Simulation (DES) is commonly used for the simulation of manufacturing systems. In many practical cases, DES practitioners have to make simplifications or to use the software in an unconventional or convoluted fashion to meet their needs. Petri nets enable the development of transparent models which allow increased flexibility and control for designers. Furthermore, Petri nets take advantage of a solid mathematical ground and constitute a simple language. However, Petri nets lack the software capabilities to realise their full potential. This study investigates the suitability and relevance of Discrete-Event Simulation (DES) software for Petri net modelling in the context of manufacturing systems. A framework is developed for the modelling of different classes of Petri nets on DES. Analytical models of asynchronous flow lines are developed. Initial results show that the analytical models are without closed-form solution and the explosion of the state space is observed, justifying the use of computational methods and simulation for the analysis of manufacturing systems. This study shows that the gain in flexibility provided by Petri nets provides a new insight into the effects of stochasticity on setup and failure times in manufacturing systems.

(Received in May 2017, accepted in November 2017. This paper was with the authors 2 months for 1 revision.)
\end{abstract}

Key Words: Petri Net, Discrete-Event Simulation, Stochastic Modelling, Manufacturing Plant Layout

\section{INTRODUCTION}

Over recent decades the capability to mathematically model stochastic systems has increased exponentially. However, many systems are still too complex to be modelled analytically or the computation of the solution is too resource intensive requiring impractical amounts of time to complete. Thus, the use of systems simulation for decision-making has become a standard in manufacturing industries and computer simulation methods are now helping practitioners to solve problems ranging from planning to new facilities design [1]. Among these simulation solutions Discrete-Event Simulation (DES) has emerged as a modelling paradigm capable of capturing a system whose state may change only at discrete points in time. It is now one of the most popular techniques in operations research [2]. DES models are usually thought of as networks of queues and servers, which translate to buffers and machines in a manufacturing context [3]. The advantages of DES are its ease of use and the resulting reduction in time required for model development. However, DES software is quite inflexible, providing only functionalities to address needs that were foreseen by its developers. In many practical cases, practitioners have to make simplifications or to use the software in an unconventional or convoluted fashion to meet their needs. Beneath the graphical user interface (GUI) DES software elements behave as black boxes that reveal little of the core functioning of the system. For instance, it is not possible to tell at first sight the difference between a machine with failures or setups or random processing times, as they all have the 
same visual appearance in DES software, yet, those features are critical to the understanding and analysis of systems.

Petri net theory has been widely developed over a period of many decades and has been used in industry for the modelling and evaluation of systems. Petri nets constitute an attractive graphically-oriented modelling framework with a strict mathematical grounding. Petri nets are concise and highly flexible with a modelling power capable of capturing most discretesystems [4]. As state based models, they are more relevant for the performance analysis of manufacturing systems than classic entity based DES models. However, Petri nets lack the software capabilities to realise their full potential and their analysis is protracted due to inherent computational complexity. This research investigates the suitability of a DES environment for the modelling of Petri nets in the context of manufacturing systems. One focus area for this work is an investigation into the fundamental functioning of different classes of Petri nets. Stochastic models of manufacturing systems are also an important area of this research as the DES Petri net models are validated against analytical models. More broadly, the study tackles the issue of simulation for the modelling of manufacturing systems and the advantages of using Petri net models over classic DES element based models. By taking advantage of the developed capabilities and advanced functions of DES software, Petri nets could be developed for the purpose of providing a more flexible and value adding solution than that realised by current DES element based models alone.

\section{RELEVANT LITERATURE}

A production line can be seen as the physical facility realising the process of transforming a variety of inputs into outputs. In manufacturing systems, this transformation is made by machines. Manufacturing a product requires a defined sequence of operations which are carried out by one or several machines [5]. Those machines can be operated manually or through automation. The state of machines in a flow line is one of idle, busy, failure, starvation or blocking [6]. Sörensen and Janssens [7] consider four states: up, down, starved or blocked. To improve the production rate, two approaches are considered: the utilisation of stand-by machines; the utilisation of buffers, with the state of the buffer being empty, full or intermediary (called 'half') [7]. Transportation means are used to move the product from one machine to the next. Capabilities in stochastic modelling of manufacturing systems have significantly expanded over the past thirty years. Stochastic models of a wide variety of types of manufacturing systems have been developed and used for practical studies. A model is defined as a representation of a system for the purpose of studying that system [8]. Two categories of system can be mentioned: mathematical and physical models. Banks et al. [2] classify simulation models as: Static vs. Dynamic; Discrete vs. Continuous; Deterministic vs. Stochastic. De Koster [8] identifies four classes of models for multi-stage lines with finite buffers, with discrete product or continuous flow, random or deterministic processing times and no failures or unreliable machines. The first recorded comprehensive treatments of stochastic models for manufacturing systems is the work from Buzacott and Shanthikumar [9]. The authors review models of flow lines, job shops, transfer lines, flexible manufacturing and assembly systems and cellular systems [9]. The work addresses the modelling of such systems, as well as the performance evaluation and issues in the control of the systems. Markovian models are especially considered in queuing theory or for asynchronous flow lines. Markov chains have been applied in manufacturing to solve a wide range of problems. Tijms [10] develop Continuous-Time Markov chains (CTMC) models for the inventory control of an inflammatory product. The problems of random demand, as used in inventory management, can be addressed by modelling as a CTMC [11]. Petri nets have since proved themselves especially useful for the modelling of manufacturing systems, in the purpose of 
control and optimisation [12]. The use of Petri nets in manufacturing applications has also received interest from various authors [13-15]. Jain et al. [13] who describe the modelling of a flexible manufacturing system. Zhang et al. [15] presented how the short-term scheduling problem of crude oil operations can be described using hybrid Petri nets while in [14], deadlock problems in Petri nets were addressed through the addition of recovery transitions. $\mathrm{Hu}$ and Zhou [16] also investigate the subject of deadlock problems within Petri nets for manufacturing applications, concluding that a new Petri net formation could be proposed that identified deadlocks in an iterative fashion within automated manufacturing systems; research in this area has also been carried out by [17]. Finally in [18] the subject of deadlock free scheduling for flexible manufacturing system is explored with future work proposed to extend research to the use of timed Petri nets, a subject explored in more detail by [19]. A consideration of constraints calculation of Petri net models for flexible manufacturing is made by [20]. In a similar vein to [20] Quintanilla et al. [21] explores Petri net use in the development service oriented holonic manufacturing systems. In work by Nie et al. [22] on Flexible Manufacturing Cells (FMC) a token oriented Petri net approach was utilised to overcome model complexities inherent in simulating FMCs. Several subclasses of Petri nets are grouped under the generic name of Ordinary Petri nets [23] and include Marked Graphs and state machine Petri nets. A marked Petri net contains tokens - graphically represented by dots - that are located in places. Their flow in the net is ruled by the transitions. When a transition is enabled, i.e. when there is the required number of tokens in each input place, the transition can fire. The firing is modelled as the removal of the tokens from the input places and the addition of tokens in the output places.

The need to develop a model taking the time of operations into consideration was first fulfilled by Ramchandani [24] when he developed timed Petri nets (TPNs), assuming that every transition would take a bounded, non-zero amount of time to fire. Timed Petri nets have a deterministic behaviour. Therefore, the state of the system i.e. the marking of the net - can be predicted at any point in time. This limitation can be sufficient in many cases. However, there is no such thing as a perfectly deterministic system in manufacturing. For this reason stochastic Petri nets (SPNs) were first described by Molloy [25, 26] who attached stochastic, exponentially distributed times to transitions. The exponential distribution has several properties that make it particularly useful for the analysis of systems [27], and describes accurately some real-life distributions, such as failure distributions. Formally, SPNs are continuous-time systems. Live and bounded SPNs are isomorphic to homogeneous Markov chains [26]. Generalised stochastic Petri nets (GSPNs) are a more powerful and flexible modelling tool than SPNs. GSPNs [28] integrate both instantaneous transitions and stochastic timed transitions. GSPNs have been widely used for the modelling of manufacturing systems. Haas and Shedler [29] have studied the modelling power of such SPN's with timed and immediate transitions and have shown that such Petri nets provide a general framework for simulation. Continuous Petri nets are the latest development of Petri nets [30]. They are obtained from discrete nets by 'fluidification' [31, 32]. The advantage of 'fluidification' originates from the extremely large state space of a plant in high-throughput context: giving a continuous approximation of a suitable discrete event system can allow the computation. Giua et al. [33] show how hybrid Petri nets, a hybrid model [34] that combines fluid and discrete event dynamics, are suited to model high-throughput production lines.

From this review of literature it has been found that there is a limit in the complexity of the Petri net models that can be developed today. Research has been conducted into methods aimed at reducing the computational effort of Petri nets analysis, though until this point DES has not been considered as a solution to address this issue. 


\section{DEVELOPMENT OF PETRI NETS IN DES}

Discrete-Event Simulation (DES) software employs a next-event technique meaning that the model is only updated when a change of the system's state is due. DES has no agreed set of standards, much less an agreed terminology [1]. Pidd [3] divides the terminology of DES into two parts: the first provides labels for the constitutive elements of a system, while the second defines how those elements engage over time. The objects in a DES modelled system are of two types: entities and resources. Entities are individual elements that are simulated. Such elements are a representation of a physical entity for example machines in a manufacturing system, or operators in a call centre. Resources are also individual elements, but they are treated as countable elements of which individual states are not tracked. Examples of resources include parts in a manufacturing system or calls in a call centre. As the simulation is run, the objects of the system, that are connected, change state. Pidd [3] uses the following terminology to describe those interactions:

- An event is an instant in time characterising a change of state in the system.

- Activities are the operations that are triggered when an event occurs. The activities are the driving force of the change in the system.

- A process is defined as a sequence of events grouped in a chronological order.

- The simulation clock is defined as the point reached by the current simulated time; this time value is then used to determine whether activities are due or not.

A wide range of DES software is available for practitioners (designers, managers etc.) to use. In this paper the selected software is WITNESS, developed by Lanner Group [35].

\subsection{Applying DES paradigm to Petri nets for manufacturing}

Standard DES models use a different paradigm than that of Petri nets. In the context of manufacturing systems, a classic DES model will model the physical elements such as instance parts, machines, buffers or labour. WITNESS uses the representation shown in Fig. 1 for those basic elements.
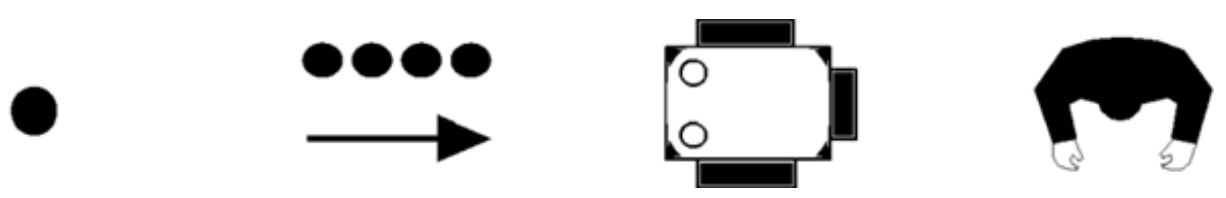

Figure 1: Basic elements in DES (from left to right: a part, a buffer, a machine and labour).

In Petri nets, while being used to model discrete event systems in a manufacturing setting [36], the paradigm is different as machines and buffers must be represented as system states. Petri nets will use places to represent the states of a machine and transitions and arcs to rule the transitions between the states, which may be used to model the behaviour of a machine. In DES a machine is modelled as an individual element containing configuration parameters. With Petri nets a combination of places and transitions is used to model machine events such as setups and breakdowns. This research utilises Ordinary/Marked Ordinary Petri nets using DES software.

\subsection{OPNs and DES for manufacturing systems}

An analogy between OPNs and DES for manufacturing systems is outlined in Table I. In WITNESS, machines pull and push parts between elements (other machines, buffer, transports etc.) according to a set of input and output rules that define the provenance, destination and quantities of the parts processed. This same functionality as that performed by arcs in Petri nets. 
Table I: Analogy between OPNs and manufacturing systems.

\begin{tabular}{|c|c|}
\hline OPNs Paradigm & DES Paradigm for Manufacturing Systems \\
\hline Place & Buffer \\
\hline Transition & Machine - general type \\
\hline Token & Part \\
\hline Arc & Machine rules \\
\hline
\end{tabular}

Simple OPNs models can be used to model basic manufacturing operations. Such models include assembly operation, transformation operation, parallel operations and disassembly/ production operations.

\subsection{Properties of stochastic Petri nets (SPNs)}

In TPNS, a real deterministic non negative integer is assigned to the transitions representing the firing time. For SPNs, there is no such thing as a firing time. Instead, a firing rate is assigned to each transition. This rate defines the amount of time that must elapse between the time the transition is enabled and the time it can be fired. In SPNs, this firing rate is a random variable with an exponential Probability Density Function (PDF). The parameter of the PDF associated with transition $i$ is $\lambda i$ and the mean firing time is $1 / \lambda i$. It means that on average, the transition fires every $1 / \lambda i$ unit of time. We will refer to stochastic timed transitions with exponentially distributed firing time as exponential transitions.

The exponential distribution is defined by its PDF:

$$
f(x)=\lambda e^{-\lambda x}, x \geq 0
$$

A random variable with such pdf is called an exponential random variable is described as a random variable that equals the distance between successive events of a Poisson process with mean $\lambda$.

For an exponential random variable:

$$
\mathbb{P}(X \leq x)=\int_{0}^{x} f(t) d t=1-e^{-\lambda x}
$$

and the mean and variance of an exponential random variable with parameter $\lambda$ are:

$$
\mu=E(X)=\frac{1}{\lambda} ; \sigma^{2}=V(X)=\frac{1}{\lambda^{2}}
$$

The definition of a firing rate results in a change in the definition of the firing of the exponential transition.

The firing of a transition is an atomic operation, meaning that the tokens are removed from the input places and added in the output places in a single operation, similarly to an instantaneous transition. The firing of an exponential transition is detailed in Fig. 2. The tokens are removed and added after the duration (random) of the transition.

SPNs are sometimes described as timed stochastic Petri nets, as if SPNs were a sub class of TPN. We can see from the firing of a SPN that this is of utmost error. In TPNs the firing is described in three steps and leaves tokens unavailable. The tokens would be input in the transition and after the duration of the transition, output. With SPNs instead, the tokens remain available, which is crucial for the modelling of conflicts situations. On DES, the equivalent firing of an exponential transition would follow the process shown in Fig. 3. 


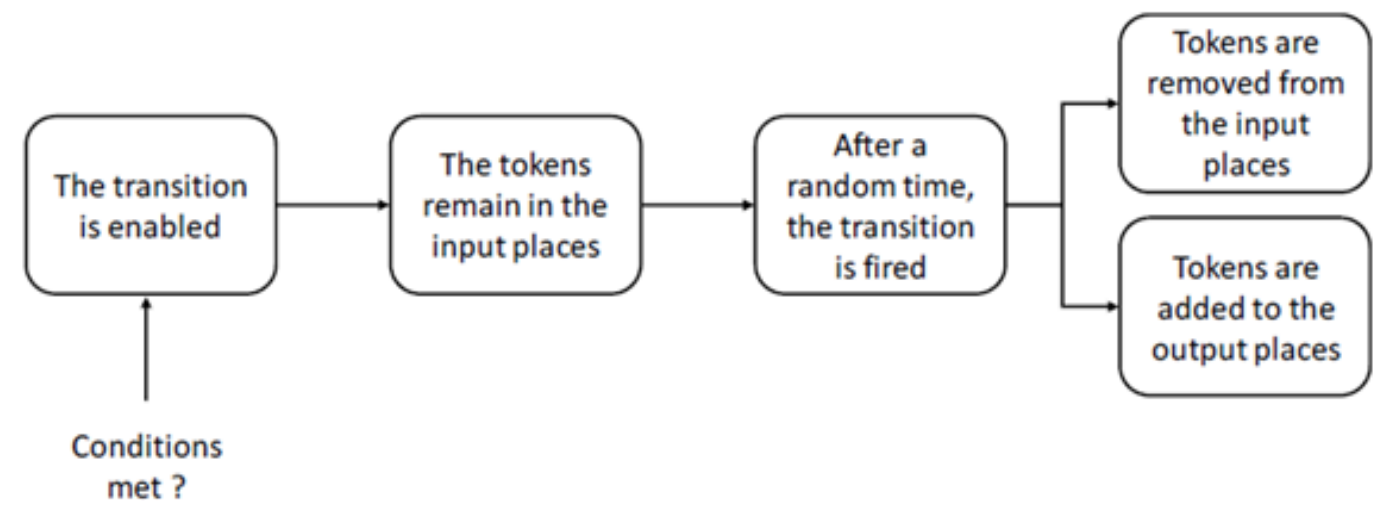

Figure 2: Theoretical firing of an exponential transition.

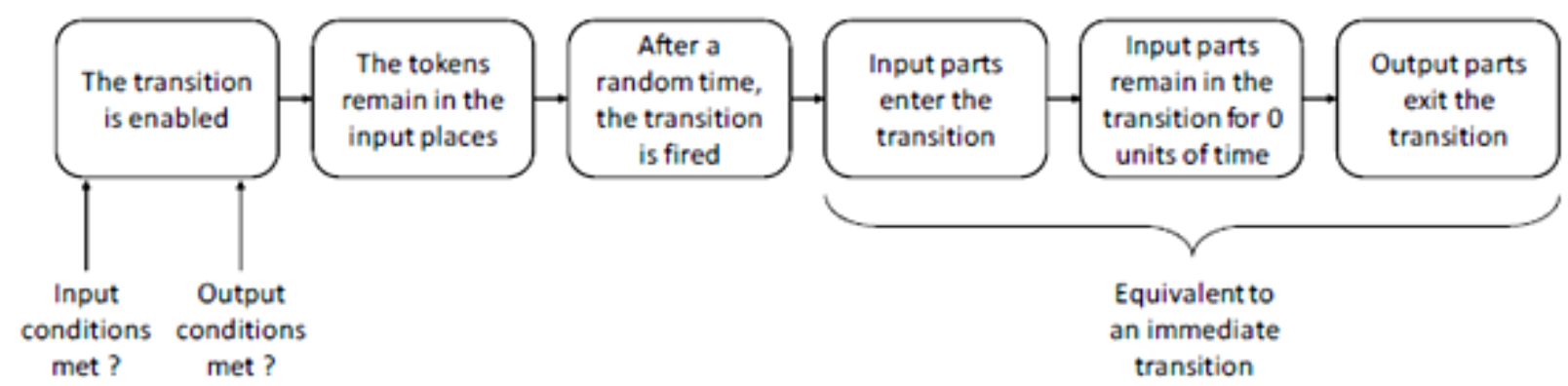

Figure 3: Firing of an exponential transition in DES.

\subsection{Modelling SPN transitions in DES}

One of the central challenges to the modelling of SPN transitions can be found in the creation of randomness within the firing. It is not possible to define that stochasticity inside the transition (machine) element by choosing a stochastic duration of the cycle time. The result would be the tokens being stored in the transition. Two solutions are considered:

- Approach 1 - Upstream generation: We create a variable to which an exponential probability density function is attached. Every time a token enters a specific place at time $t$, the variable takes a value $v$ from the exponential distribution. When the time is superior or equal to $t+v$ the transition fires like an immediate transition.

- Approach 2 - Statistical test: At every defined time interval (as small as possible), a statistical test is carried out to decide whether or not the transition has fired. This method is based on survival theory and the memoryless property of the exponential distribution.

The properties of those solutions are evaluated in the modelling of a conflicting SPN. Such situation is represented in Fig. 4. In Fig. 4, a token is in $p_{1}$ and both $t_{1}$ and $t_{2}$ are enabled. Because of the stochasticity, we do not know which transition is going to fire first. That is why is it pivotal to keep the token available in $p_{1}$ after the transitions are enabled, to leave the possibility to both transitions to fire. We now introduce the following elements:

- $\lambda_{1}$ the firing rate of $t_{1}$,

- $\lambda_{2}$ the firing rate of $t_{2}$.

Through experimentation it has been found that the first approach (upstream generation) is preferred due to its performance and simplicity in implementation.

\subsection{Generalised stochastic Petri nets}

GSPNs are adapted to the modelling of manufacturing systems. The modelling of GSPNs on DES is straightforward once OPNs and SPNs have been modelled. Indeed, GSPNs simply contain both immediate and exponential transitions. In this section two Petri net models of 
manufacturing systems are designed and modelled, these will be used in section 6 to validate the behaviour of DES Petri nets against the behaviour of equivalent analytical models. These two models are a single failure-prone machine and an asynchronous two-machine one-buffer transfer line. Fig. 5 shows the GSPN model of a machine with failures [31]. In our case, we will consider failures happening during the processing. The net is a mapping of the state of the machine and of the transition between those states. For a machine with failures, four states are considered:

- Machine up and busy (processing),

- Machine down (waiting for repair),

- Machine up and idle (blocked),

- Machine up and free (starving).

In Fig. 5 a model of a machine with failures is represented.

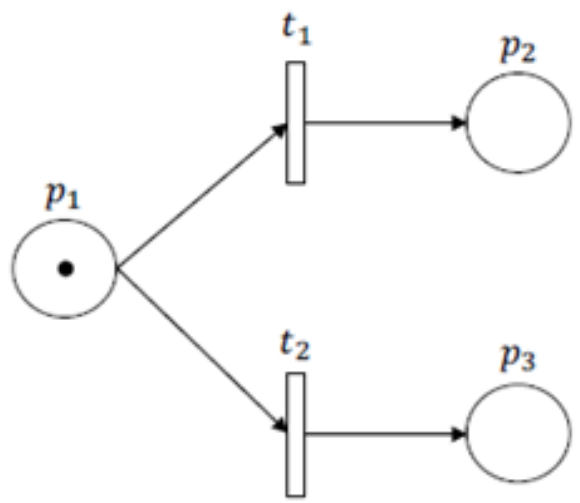

Figure 4: Conflicting SPN.

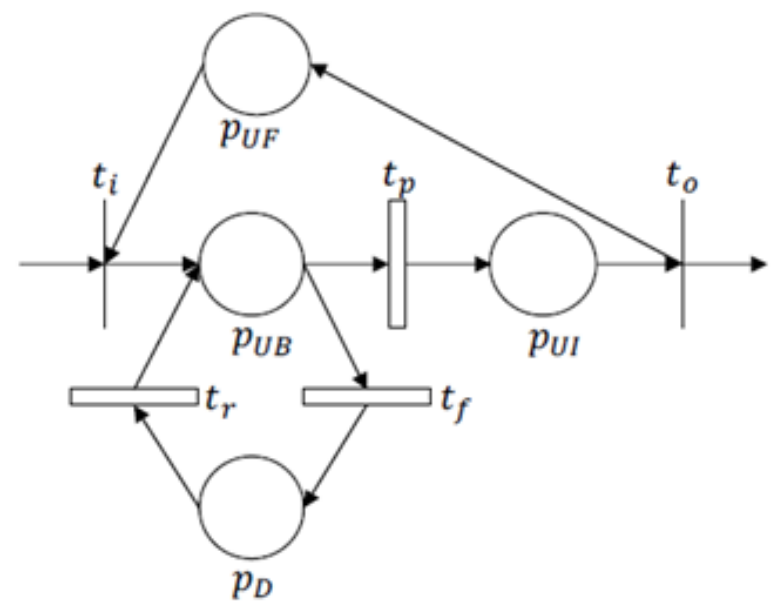

Figure 5: Machine with failure module.

In the following, we will consider the same machine with failures and add it setups. This machine is created by adding a place $P S U$ before $P U B$ in which a token stays until $t s$ is fired at a rate $\lambda s$ which is the setup rate.

\subsection{Two machines one buffer transfer line}

The two machines one buffer system is based on the single machine model. The principle is to connect two single machine blocks by a place representing a buffer. Because this system is similar to the single machine, the modelling in WITNESS is equivalent.

\section{MODEL COMPARISON}

In this section of the paper the behaviour of the DES Petri net single and two machine systems is evaluated. For each system, three models are compared: the DES Petri net model, the analytical model and a classic DES element based model. For each of those models, equivalent performance metrics are developed for comparison. The experiments are run according to Table II. In each scenario one parameter is defined as a variable.

It is reminded that we note:

- the rate of repair,

- the rate of failure,

- the rate of process,

- the rate of setup. 
Table II: One parameter analysis for the single machine model.

\begin{tabular}{|c|c|c|c|c|}
\hline Experiment & Setup rate $\boldsymbol{d}$ & Repair rate $\boldsymbol{a}$ & Process rate $\boldsymbol{c}$ & Failure rate $\boldsymbol{b}$ \\
\hline $\mathbf{1}$ & Variable & 0.1 & 0.25 & 0.025 \\
\hline $\mathbf{2}$ & 0.15 & Variable & 0.25 & 0.025 \\
\hline $\mathbf{3}$ & 0.15 & 0.1 & Variable & 0.025 \\
\hline $\mathbf{4}$ & 0.15 & 0.1 & 0.25 & Variable \\
\hline
\end{tabular}

For each experiment the steady state probabilities will be used as standard performance metrics along with the following notations:

- P1 is the probability for the machine to be down, calculated for the analytical model. P1_PN specifies that this is calculated for the DES Petri net model. P1_W specifies that this is calculated for the classic DES model.

- P2 is the probability for the machine to be processing, calculated for the analytical model P2_PN specifies that this is calculated for the DES Petri net model. P2_W specifies that this is calculated for the classic DES model.

- P3 is the probability for the machine to be in setup, calculated for the analytical model. P3_PN specifies that this is calculated for the DES Petri net model. P3_W specifies that this is calculated for the classic DES model.

For this section, the comparison will be developed on a logarithmic scale spanning from -0.5 to 0 . The first observation is that these graphs roughly superimpose themselves, meaning that the models have the same behaviour. The analytical model is by definition theoretical and thus the model of reference. The deviation of the DES Petri net model from this reference is computed in Fig. 6. We observe that the error remains between 0.015 and -0.015 , and the probabilities are all within the similar error range.

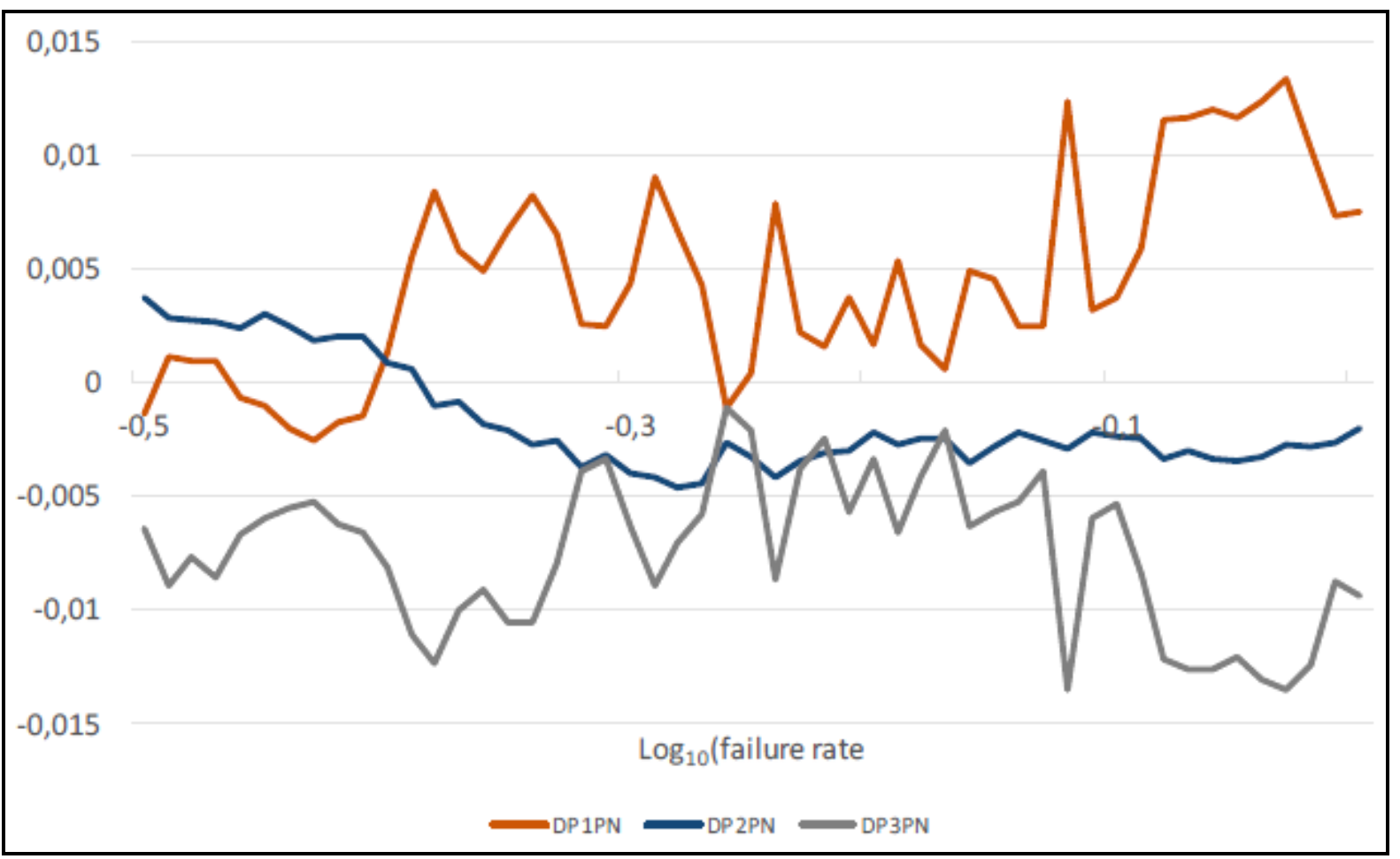

Figure 6: Error between theoretical and DES Petri net models.

A metric complementary to the absolute error is the percent error. The percent error is calculated as: 


$$
\% \text { error }=\frac{\text { Theoretical Value }- \text { Empirical value }}{\text { Theoretical Value }}
$$

That percentage of error is represented in Fig. 7. The deviation from the reference is spanning from $+10 \%$ to $-10 \%$, which is enough to validate the model. However, this is also to be put in perspective with the absolute value of the error. For instance, P1 is taking values very close from 0 and WITNESS results have only three decimals, thus the error can only increase as the accuracy required increases.

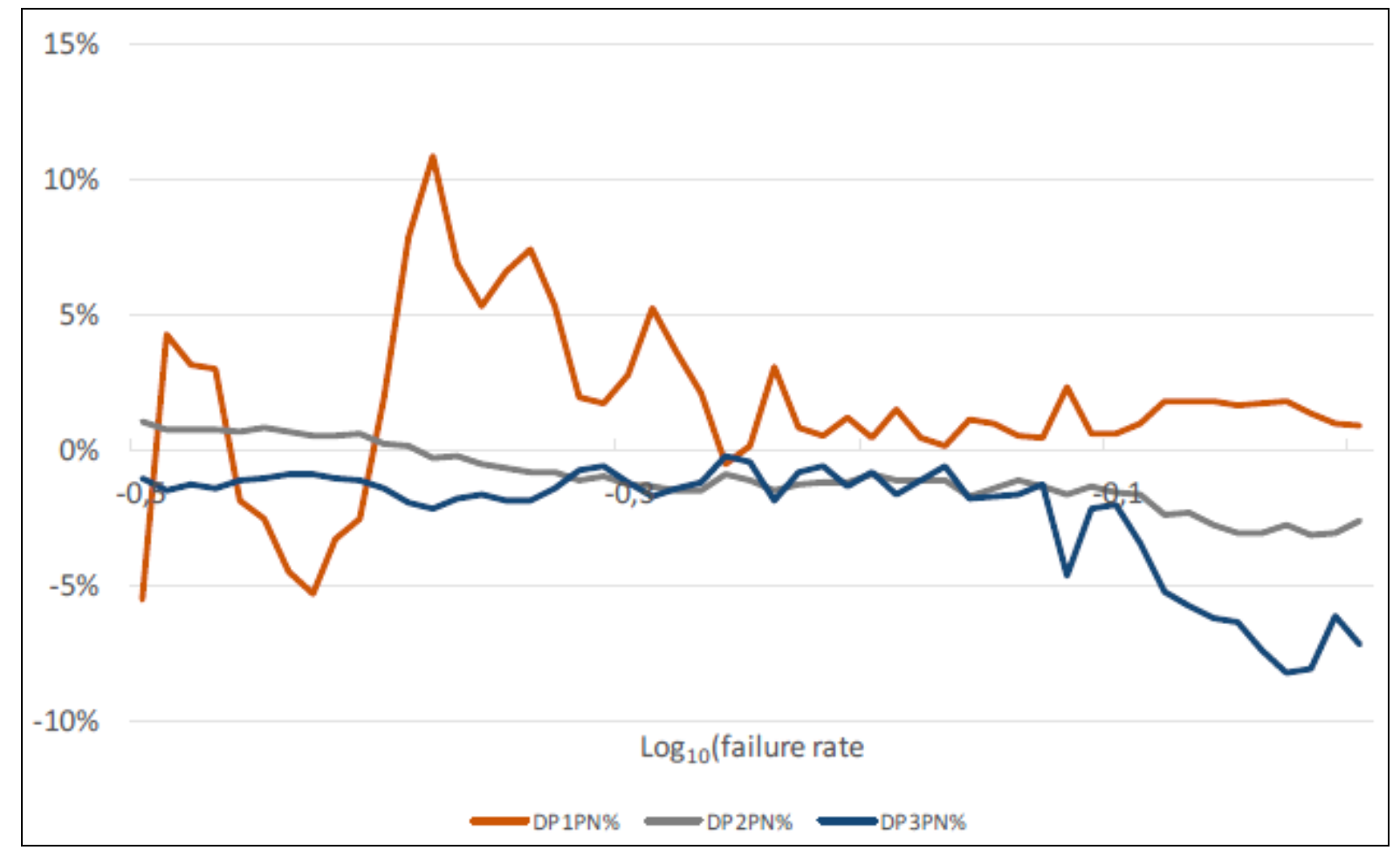

Figure 7: Percent error between theoretical and DES Petri net models.

As this study proposes DES Petri net models as an alternative to classic DES models, a comparison has to be made between those two model types, independently from the reference results. For this experiment, the Petri net model is proved to behave equivalently if not better than the classic DES model, as shown in Fig. 8.

Expert opinion was sought to provide feedback on DES Petri model use. The main advantage identified was the increased modelling power that would result from using Petri nets. Petri nets are not as restrained by the software as standard DES models are. WITNESS has only ten main states to describe a machine, but in case of modelling a robot, a greater number of states would be required. Petri nets are by definition state based. Therefore, the models are not limited and allow a customised approach for the state modelling and analysis. The flexibility afforded by Petri nets through their use as a low level language was also noted. A major opportunity was identified in the modelling of Petri nets in DES, the use of a modular approach to model development. By defining the single machine model as a module, it would take no more time to create a DES Petri net model than it would take to create a standard DES model. 


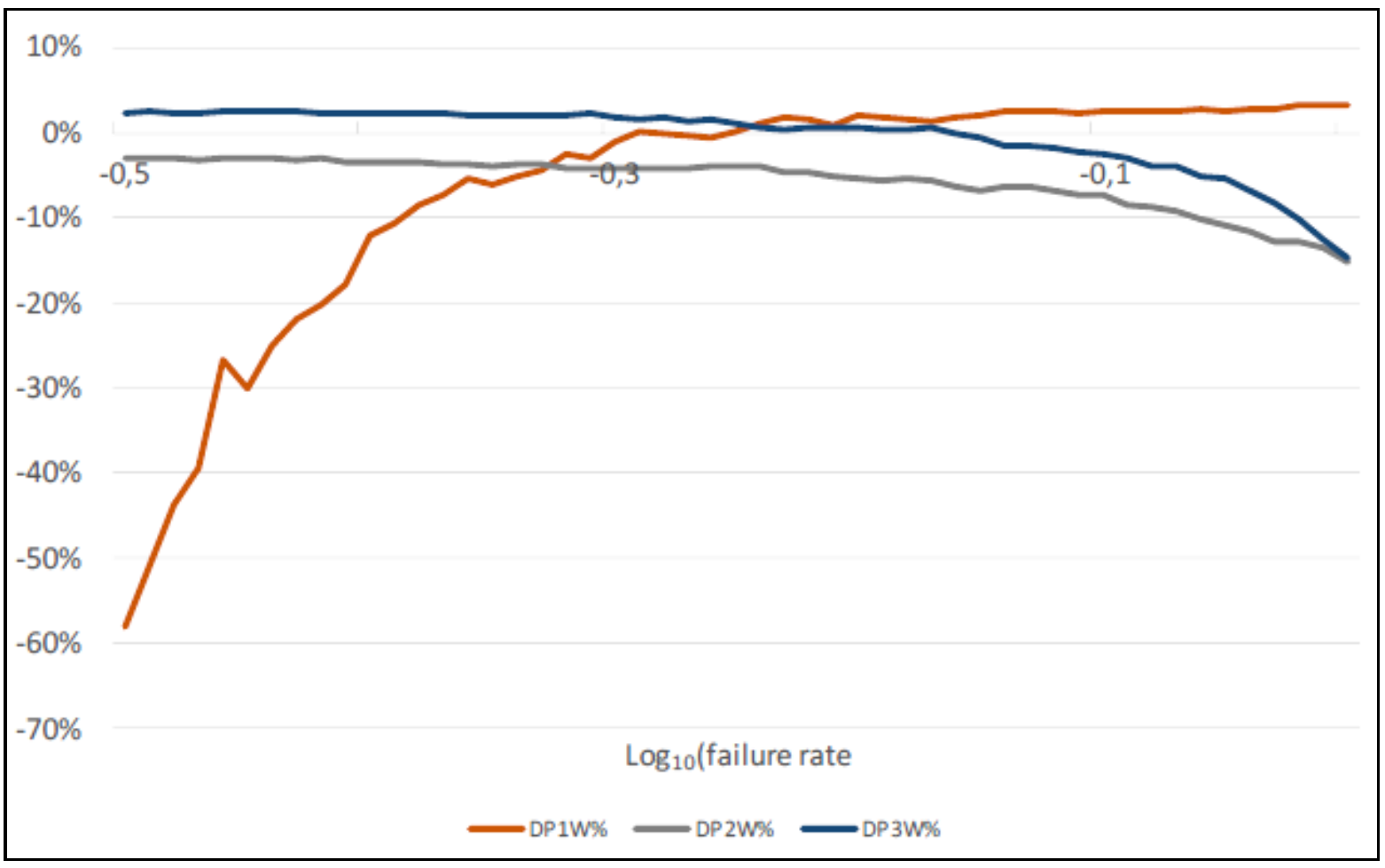

Figure 8: Percent error between standard DES Petri net and classic DES models.

\section{CONCLUSION}

A detailed analysis of both DES and Petri net paradigms has been developed. The outcome of this analysis is a framework allowing the translation of Ordinary Petri nets into DES element based models. Using the original definition given in the work of Ramchandani [24], timed Petri nets were developed in a DES environment. As such models are deterministic, stochastic models of Petri nets were then introduced with stochastic Petri nets. Two approaches were considered for the modelling of stochastic transitions, both were compared and the most promising one was chosen. To validate the framework developed for the modelling of Petri nets two application cases were proposed. The first involved the modelling and analysis of a single machine with setups and failures. The second modelled a two machine one buffer asynchronous flow line, using machines with setups and failures. The two systems were then developed in a DES environment. To verify the accuracy of the models of DES Petri nets developed within the DES framework, two other equivalent models were created. Classic element based DES models were developed following the standard use of DES environments. Secondly, a theoretical model was set, using stochastic processes theory.

The performance metrics of the three models have been compared for each system. Several experiments were carried out to obtain the steady state probabilities as a function the parameters of the model. For every parameter, the DES Petri net model was proved to have the same behaviour as the theoretical reference model. More, the DES Petri net models were shown to behave an equivalent way to classic DES models, if not better. These findings were then discussed and validated with a simulation expert. The validation highlighted the advantages of using DES Petri nets as a modelling framework, including increased flexibility, modelling power and control. Two main limitations to this approach have been found during this research. When using DES for the modelling of Petri nets the computational effort required to run such simulations is substantial. This could be an issue when running complex models. A second limitation lies in the ease of use of Petri net models. Classic element based models have the advantage in being intuitive, if not natural to use. Thus, a gap is identified 
between the current common knowledge of practitioners in the simulation of systems and the use of Petri net theory. It is also the case that the use of Petri nets in industry is relatively sparse [37]. The findings of the study can be used as a base for future research involving both Petri nets and simulations. The authors identified three major axes of improvements to the theory developed in the study. The first is to increase the complexity of the models. So far, only simple models have been evaluated. The development of more complex models would allow for the qualification of potential drawbacks of using Petri nets (through longer modelling time and an increased size of the models). In future work the framework will be extended to model other classes of Petri nets. Extended stochastic Petri nets are a good candidate and would considerably increase the modelling power of the Petri net models, allowing the description of complex behaviours. The theory of continuous Petri nets has been mentioned in this study and constitutes a whole new paradigm through which DES could be extended.

\section{ACKNOWLEDGEMENTS}

This work was supported by Innovate UK (project ref. 101888) and the Engineering and Physical Sciences Council (EPSRC) (grant no. EP/M506813/1) (Towards Zero Prototyping of Factory Layouts and Operations Using Novel Gaming and Immersive Technologies).

\section{REFERENCES}

[1] Robinson, S. (2003). Simulation: The Practice of Model Development and Use, John Wiley and Sons Ltd., Chichester

[2] Banks, J.; Carson, J. S.; Nelson, B. L.; Nicol, D. M. (2010). Discrete-Event System Simulation, $5^{\text {th }}$ ed., Prentice Hall, Upper Saddle River

[3] Pidd, M. (2004). Computer Simulation in Management Science, $5^{\text {th }}$ ed., John Wiley and Sons Ltd., Chichester

[4] Murata, T. (1989). Petri nets: properties, analysis and applications, Proceedings of the IEEE, Vol. 77, No. 4, 541-580, doi: $10.1109 / 5.24143$

[5] Proth, J.-M.; Xie, X. (1996). Petri Nets - A Tool for Design and Management of Manufacturing Systems, John Wiley and Sons Ltd., Chichester

[6] Liu, C.; Shi, H.; Yuan, J. (2008). Modeling and performance analysis of flow lines with stochastic failures based on Petri nets, Proceedings of the $7^{\text {th }}$ World Congress on Intelligent Control and Automation, Chongqing, 6439-6444, doi:10.1109/WCICA.2008.4593904

[7] Sörensen, K.; Janssens, G. K. (2014). A Petri net model of a continuous flow transfer line with unreliable machines, European Journal of Operational Research, Vol. 152, No. 1, 248-262, doi:10.1016/S0377-2217(02)00653-7

[8] De Koster, M. B. M. (1989). Capacity Oriented Analysis and Design of Production Systems, Lecture Notes in Economics and Mathematical Systems, No. 323, Springer-Verlag, Berlin

[9] Buzacott, J. A.; Shanthikumar, J. G. (1993). Stochastic Models of Manufacturing Systems, Prentice-Hall, Englewood Cliffs

[10] Tijms, H. C. (2003). A First Course in Stochastic Models, Wiley, Chichester

[11] Yin, K. K.; Liu, H.; Yin, G. G. (2003). Stochastic models and numerical solutions for production planning with applications to the paper industry, Computers \& Chemical Engineering, Vol. 27, No. 11, 1693-1706, doi:10.1016/S0098-1354(03)00136-4

[12] Al-Jaar, R. Y.; Desrochers, A. A. (1990). Performance evaluation of automated manufacturing systems using generalized stochastic Petri nets, IEEE Transactions on Robotics and Automation, Vol. 6, No. 6, 621-639, doi:10.1109/70.63259

[13] Jain, A.; Jain, P. K.; Singh, I. P. (2006). Performance modeling of FMS with flexible process plans - a Petri net approach, International Journal of Simulation Modelling, Vol. 5, No. 3, 101113, doi:10.2507/IJSIMM05(3)2.064 
[14] Chen, Y. F.; Li, Z. W.; Al-Ahmari, A.; Wu, N. Q.; Qu, T. (2017). Deadlock recovery for flexible manufacturing systems modeled with Petri nets, Information Sciences, Vol. 381, 290-303, doi:10.1016/j.ins.2016.11.011

[15] Zhang, S. W.; Wu, N. Q.; Li, Z. W.; Qu, T.; Li, C. D. (2017). Petri net-based approach to shortterm scheduling of crude oil operations with less tank requirement, Information Sciences, Vol. 417, 247-261, doi:10.1016/j.ins.2017.07.009

[16] Hu, H.; Zhou, M. C. (2015). A Petri net-based discrete-event control of automated manufacturing systems with assembly operations, IEEE Transactions on Control Systems Technology, Vol. 23, No. 2, 513-524, doi:10.1109/TCST.2014.2342664

[17] Liu, H.; Xing, K.; Zhou, M. C.; Han, L.; Wang, F. (2014). Transition cover-based design of Petri net controllers for automated manufacturing systems, IEEE Transactions on Systems, Man, and Cybernetics: Systems, Vol. 44, No. 2, 196-208, doi:10.1109/TSMC.2013.2238923

[18] Lefebvre, D. (2016). Deadlock-free scheduling for flexible manufacturing systems using untimed Petri nets and model predictive control, IFAC-PapersOnLine, Vol. 49, No. 12, 384-389, doi:10.1016/j.ifacol.2016.07.635

[19] Başak, Ö.; Albayrak, Y. E. (2015). Petri net based decision system modeling in real-time scheduling and control of flexible automotive manufacturing systems, Computers \& Industrial Engineering, Vol. 86, 116-126, doi:10.1016/j.cie.2014.09.024

[20] Chen, Y. F.; Li, Z. W.; Zhou, M. C. (2014). Optimal supervisory control of flexible manufacturing systems by Petri nets: A set classification approach, IEEE Transactions on Automation Science and Engineering, Vol. 11, No. 2, 549-563, doi:10.1109/TASE.2013.2241762

[21] Quintanilla, F. G.; Cardin, O.; L'Anton, A.; Castagna, P. (2016). A Petri net-based methodology to increase flexibility in service-oriented holonic manufacturing systems, Computers in Industry, Vol. 76, 53-68, doi:10.1016/j.compind.2015.09.002

[22] Nie, X. D.; Chen, X. D; Chen, X. (2016). Simulation study of flexible manufacturing cell based on token-oriented Petri net model, International Journal of Simulation Modelling, Vol. 15, No. 3, 566-576, doi:10.2507/IJSIMM15(3)CO14

[23] Desrochers, A. A.; Al-Jaar, R. Y. (1995). Applications of Petri Nets in Manufacturing Systems Modeling, Control and Performance Analysis, IEEE Press, New York

[24] Ramchandani, C. (1973). Analysis of Asynchronous Concurrent Systems by Timed Petri Nets, $\mathrm{PhD}$ Thesis, Massachusetts Institute of Technology, Cambridge

[25] Molloy, M. K. (1981). On the Integration of Delay and Throughput Measures in Distributed Processing Models, PhD Thesis, University of California, Los Angeles

[26] Molloy, M. K. (1982). Performance analysis using stochastic Petri nets, IEEE Transactions on Computers, Vol. C-31, No. 9, 913-917, doi:10.1109/TC.1982.1676110

[27] Hatono, I.; Yamagata, K.; Tamura, H. (1991). Modeling and online scheduling of flexible manufacturing systems using stochastic Petri nets, IEEE Transactions on Software Engineering, Vol. 17, No. 2, 126-132, doi:10.1109/32.67588

[28] Ajmone Marsan, M.; Conte, G.; Balbo, G. (1984). Class of generalised stochastic Petri nets for the performance evaluation of multiprocessor systems, ACM Transactions on Computer Systems, Vol. 2, No. 2, 93-122, doi:10.1145/190.191

[29] Haas, P. J.; Shedler, G. S. (1989). Stochastic Petri net representation of discrete event simulations, IEEE Transactions on Software Engineering, Vol. 15, No. 4, 381-393, doi:10.1109/ 32.16599

[30] Di Febbraro, A.; Giua, A.; Menga, G. (2001). Guest Editorial for the special issue on Hybrid Petri Nets, Discrete Event Dynamic Systems, Vol. 11, No. 1-2, 5-8

[31] Alla, H.; David, R. (1998). A modelling and analysis tool for discrete events systems: continuous Petri net, Performance Evaluation, Vol. 33, No. 3, 175-199, doi:10.1016/S0166-5316(98)00016-9

[32] Silva, M.; Recalde, L. (2002). Petri nets and integrality relaxations: A view of continuous Petri net models, IEEE Transactions on Systems, Man and Cybernetics - Part C: Applications and Reviews, Vol. 32, No. 4, 314-327, doi:10.1109/TSMCC.2002.806063

[33] Giua, A.; Pilloni, M. T.; Seatzu, C. (2005). Modelling and simulation of a bottling plant using hybrid Petri nets, International Journal of Production Research, Vol. 43, No. 7, 1375-1395, doi: $10.1080 / 00207540412331299602$ 
[34] Benvenuti, L.; Farina, L. (2002). Positive and compartmental systems, IEEE Transactions on Automatic Control, Vol. 47, No. 2, 370-373, doi:10.1109/9.983382

[35] Lanner Group. Witness Horizon, https://www.lanner.com/technology/witness-simulationsoftware.html, accessed on 09-05-2017

[36] Li, Z.; Xu, L. D. (2003). Polychromatic sets and its application in simulating complex objects and systems, Computers \& Operations Research, Vol. 30, No. 6, 851-860, doi:10.1016/S03050548(02)00038-2

[37] Vergidis, K.; Turner, C. J.; Tiwari, A. (2008). Business process perspectives: Theoretical developments vs. real-world practice, International Journal of Production Economics, Vol. 114, No. 1, 91-104, doi:10.1016/j.ijpe.2007.12.009 